\title{
Wavelet Bases Adapted to Inhomogeneous Cases
}

\author{
Pieter W. Hemker and Frédérique Plantevin
}

\begin{abstract}
In this chapter we describe a general strategy for the construction of wavelet bases when the loss of invariance under translation prevents the use of Fourier techniques.
\end{abstract}

\section{$\S 1$ Introduction}

The construction of wavelet bases in the usual case of $L^{2}(\mathbb{R})$ is based on the use of Fourier techniques, i.e., on the invariance under translation. However, in many practical cases, this invariance does not hold and one has to look for an other strategy. Such a new approach has been performed and used for the following examples:

- Wavelet basis of $L^{2}(\Omega)$, where $\Omega$ is an arbitrary open set of $\mathbb{R}^{n}$, with Dirichlet boundary conditions [8];

- Wavelet basis of $L^{2}(I)$, where $I$ is an interval of $\mathbb{R}$, without any boundary condition $[5,10]$, or with arbitrary boundary conditions $[1,2]$;

- Wavelets adapted to the differential operator $T=D^{\star} a D$, where $a$ is the operator of pointwise multiplication by the complex-valued function $a(x)$ which is not regular and which satisfies

$$
|a(x)| \leq M=\|a\|_{\infty} \text { and } \operatorname{Re} a(x) \geq 1 \text { a.e. }
$$

(the last property means that $a(x)$ is accretive);

$D$ is the operator $-i \frac{d}{d x}$ on the domain $H^{1}(\mathbb{R})[3]$. 
- Wavelets adapted to the "same" operator $T$ where $\mathbb{R}$ is replaced by an interval $I \subset \mathbb{R}$ for which arbitrary boundary conditions are added. The domain $V$ of $D$ is now a closed subspace of $H^{1}(I)$ which "contains" the boundary conditions, and the domain $V^{\star}$ of its adjoint $D^{\star}$ is an other closed subspace of $H^{1}(I)$ which "contains" the complementary boundary conditions, [4];

- Wavelets associated with a family of irregular meshes [13].

Our aim is to present the generic construction of wavelet bases adapted to inhomogeneous cases, that is common to the treatment of most of the above examples. As an introduction, we will recall a number of basic ideas and definitions used in the homogeneous case, i.e., in the case of $L^{2}(\mathbb{R})$. This will allow us to show where the differences start.

The multiresolution analysis, as it was defined by Meyer and Mallat [11] for $L^{2}(\mathbb{R})$, provides the general algorithm for the construction of wavelet bases for $L^{2}(\mathbb{R})$.

We recall that a multiresolution analysis of $L^{2}(\mathbb{R})$ consists of a sequence of nested closed subspaces $\left(V_{j}\right)_{j \in \mathbb{Z}}$ of $L^{2}(\mathbb{R})$ which allow to approximate any squareintegrable function with an increasing accuracy :

$$
V_{j} \subset V_{j+1}, \quad \bigcap_{j \in \mathbb{Z}} V_{j}=\{0\} \text { and } \overline{\bigcup_{j \in \mathbb{Z}} V_{j}}=L^{2}(\mathbb{R}) .
$$

Each $V_{j}$ is invariant under dyadic translation:

$$
\forall k \in \mathbb{Z} \quad f(x) \in V_{j} \quad \Longleftrightarrow \quad f\left(x-k 2^{-j}\right) \in V_{j} .
$$

The spaces $V_{j}$ are derived from each other by scaling:

$$
f(x) \in V_{j} \Longleftrightarrow f(2 x) \in V_{j+1} .
$$

And finally, there exists a regular and well localised function $g(x)$ in $V_{0}$ such that the set $(g(x-k))_{k \in \mathbb{Z}}$ forms a Riesz basis of $V_{0}$. The regularity $r, r \in \mathbb{N}$, and the localisation of the function $g(x)$ are expressed by

$$
\left\{\begin{array}{l}
\text { (i) } g \in C^{r-1} \\
\text { (ii) } g^{(r)} \text { exists almost everywhere; } \\
\text { (iii) } \forall m, 0 \leq m \leq r, \forall p \exists C_{m, p}\left|g^{(m)}(x)\right| \leq C_{m, p}(1+|x|)^{-p} .
\end{array}\right.
$$

We say that the so-defined multiresolution analysis is $r$-regular.

We see that this definition requires, among other properties, invariance under translation of the approximation spaces $V_{j}$. If we have functions restricted to a finite interval $I$, then the end points of the interval create an inhomogeneity which breaks the invariance under translation: when we want to construct a wavelet basis 
for $L^{2}(I)$, the multiresolution analysis must first be redefined. It is by far not the only example where the usual definition of multiresolution analysis is not suitable. The next section is devoted to the description of three of the above mentioned inhomogeneous cases.

Let us first return to the construction of wavelet bases in the classical case of $L^{2}(\mathbb{R})$. As we know, it starts by considering the subspace $W_{j}$, orthogonal to $V_{j}$ in $V_{j+1}$. The fundamental theorem proves the existence of a function $\psi \in W_{0}$ which has the same decay and regularity properties as $g$ and of which all translated copies (translated by integers) form an orthonormal basis of $W_{0}$. By scaling, one derives immediately that the functions $\psi_{j k}(x)=2^{\frac{j}{2}} \psi\left(2^{j} x-k\right)$, with $k \in \mathbb{Z}$, form an orthonormal basis of $W_{j}$. Further, by construction we have

$$
L^{2}=\underset{j \in \mathbb{Z}}{\oplus} W_{j}
$$

Since the sum is orthogonal, the family $\left(\psi_{j k}\right)_{j, k \in \mathbb{Z}}$ is an orthonormal basis of $L^{2}(\mathbb{R})$ and for any function $f \in L^{2}(\mathbb{R})$ we have

$$
\begin{gathered}
f=\sum_{j, k \in \mathbb{Z}} d_{j k} \psi_{j k}, \quad \text { where } d_{j k}=\left\langle f, \psi_{j k}\right\rangle, \\
\text { and } \int|f|^{2}=\sum_{j, k \in \mathbb{Z}}\left|d_{j k}\right|^{2} .
\end{gathered}
$$

In practical situations, one starts at a coarsest level $j_{0}$ and one considers only $j \geq j_{0}$. Thus, one uses the equality

$$
L^{2}=V_{j_{0}} \oplus\left(\underset{j=j_{0}}{\oplus} W_{j}\right)
$$

which gives

$$
f(x)=\sum_{k \in \mathbb{Z}} c_{j_{0} k} g_{j_{0} k}(x)+\sum_{k \in \mathbb{Z}, j \geq j_{0}} d_{j k} \psi_{j k}(x) .
$$

The first sum describes the projection of $f$ on $V_{j_{0}}$, i.e., the approximation of $f$ on the coarsest level, and the decomposition of $f$ on each $W_{j}$, the missing details between two successive levels of approximation. Here we do not have equality of the norms anymore but only equivalence, i.e., there exists two positive constants $A$ and $B$, not depending on $j_{0}$, such that

$$
A \int|f|^{2} \leq \sum_{k \in \mathbb{Z}}\left|c_{j_{0} k}\right|^{2}+\sum_{k \in \mathbb{Z}, j \geq j_{0}}\left|d_{j k}\right|^{2} \leq B \int|f|^{2},
$$


which is also written as

$$
\int|f|^{2} \sim \sum_{k \in \mathbb{Z}}\left|c_{j o}\right|^{2}+\sum_{k \in \mathbb{Z}, j \geq j_{0}}\left|d_{j k}\right|^{2} .
$$

Most important is the unconditional convergence of the series because it means that the associated algorithms for analysis and synthesis are stable (the constants $A$ and $B$ do not depend on $j_{0}$ ). Although orthonormal bases may be convenient, they are not necessary for the practical use as long as the stability of the associated algorithms is preserved. This is why one may use unconditional but nonorthogonal bases. This choice means that, instead of each $W_{j}$, one will consider a nonorthogonal complementary set of $V_{j}$ in $V_{j+1}$, leading to another decomposition of $L^{2}(\mathbb{R})$ :

$$
L^{2}(\mathbb{R})=\underset{j \in \mathbb{Z}}{\oplus} X_{j}
$$

or

$$
L^{2}(\mathbb{R})=V_{j 0} \oplus\left(\underset{j \geq j_{0}}{\oplus} X_{j}\right)
$$

where the sums are not orthogonal but only direct. We emphasise that the set of functions that form the bases of the spaces $X_{j}$ does not directly form a basis of $L^{2}(\mathbb{R})$. This fact must now be proved in order to make (4) or (5) hold. We shall see that these oblique spaces are constructed by hand, and their bases are explicitly given by the construction. Thus, on the one hand we have the standard wavelet bases for the $W_{j}$ spaces which trivially form a basis of $L^{2}(\mathbb{R})$ but are difficult to compute, and on the other hand we have explicit bases of the oblique $X_{j}$ spaces, for which the property that they form a basis of $L^{2}(\mathbb{R})$ requires a specific proof. The second approach is clearly better adapted to numerical purposes and indeed used more and more.

Let us recall that, in order to prove the existence of an orthonormal basis of $W_{0}$ and to effectively construct it in the translation invariant case, the essential idea is to systematically work on the Fourier side. This is no longer possible in the inhomogeneous cases. However, we may still have access to the oblique complement $X_{j}$, of $V_{j}$ in $V_{j+1}$ and this allows us to construct $W_{j}$ and its basis. More precisely, we can construct multiresolution analyses in such a way that an oblique complement of $V_{j}$ in $V_{j+1}$ is always accessible. Finally, the bases of the $X_{j}$ spaces are interesting for themselves. We have already mentioned their numerical simplicity. In addition, by a suitable definition of $X_{j}$ we construct a basis that is adapted to different situations. This procedure gives us some additional freedom.

We now have shown the strategy of the construction: a redefinition of the multiresolution analysis and the construction of oblique complementary sets $X_{j}$ of $V_{j}$ in $V_{j+1}$. Then we shall need specific mathematical tools to prove that the bases of these $X_{j}$ spaces form an unconditional basis of $\overline{U_{j \in \mathbb{Z}} V_{j}}$. 
The goal of this paper is not to show all the results and the details of the construction which one can find in the above mentioned references. We shall also willingly omit some aspects of motivation when they divert too far from our central point. Neither do we pretend to give an exhaustive overview of the results in this field of research (which is in rapid development) not even, in the above mentioned references. Our aim is to show the common skeleton of the construction of wavelet bases through three different examples which we assume to be representative of our purpose. These examples (1, a special case of 4 and 5 in the list above) are described in the next section. In the third section, we shall show the definition of the multiresolution analyses associated to them. Once the multiresolution analysis is given, the construction of wavelets may start. This is what is done in the section 4, while the section 5 is devoted to the mathematical tools which are needed to prove that the constructed wavelets form a Riesz basis of $\underset{j}{\cup} V_{j}$ indeed. In these three sections, the approach is general and followed by the application to the specific examples.

\section{§2 Examples of inhomogeneous problems}

We denote by $\Lambda$ the set of the wavelet labels. $\Lambda$ is the set of all the numbers $\lambda=\left(k+\frac{1}{2}\right) 2^{-j}$ which have a one-to-one correspondence with the pairs $\left(k 2^{-j}, 2^{-j}\right)$, i.e., points in the position-scale plane. In the $L^{2}(\mathbb{R})$ case, both $j$ and $k$ are taken from $\mathbb{Z}$, or if we choose the decomposition (1.3), $k \in \mathbb{Z}$ and $j \geq j_{0}$. This expresses the fact that the union of all dyadic numbers is dense in $\mathbb{R}$. It can easily be generalised to $\mathbb{R}^{n}$ by considering $k \in \mathbb{Z}^{n}$ and $j \in \mathbb{Z}$. When one considers an interval or a fortiori an irregular mesh, $k$ and $j$ will be taken only from a part of $\mathbb{Z}$. For these cases, $\Lambda$ will be precisely defined later. For the present time, the wavelets are labeled by $\lambda \in \Lambda$ which we assume to be adapted to each particular case. In the examples below, we call $2^{-j o}$ the largest scale taken in account. This implies that we shall consider decompositions of type (1.3) and (1.5).

Let us present the three examples.

The first is the construction of an orthonormal wavelet basis of $L^{2}(\Omega)$, where $\Omega$ is an arbitrary open set of $\mathbb{R}^{n}$, with homogeneous Dirichlet boundary conditions. The wavelets will belong to $C_{0}^{2 m-2}(\Omega), m \geq 1$, i.e., they will be of regularity $C^{2 m-2}$ with support included in $\Omega$ (the last property reflects the Dirichlet conditions). In [8], these wavelets are used to analyze the Hölder and Sobolev spaces, $C_{0}^{r}(\Omega)$ and $H_{0}^{s}(\Omega), 0<r<2 m-2$ and $0<s<2 m-2$. Briefly, this means that the size of the wavelet coefficients, $\left\langle f, \psi_{\lambda}\right\rangle$, of $f \in L^{2}(\Omega)$ enables us to measure the degree of regularity of $f$ (see [11] chapter VI). In what follows, we shall not describe how this is done. However, this motivation is of importance because it sets a condition to how the multiresolution analysis is constructed. Actually, the key of the characterisation of Hölder spaces by means of wavelet bases lies in the cancellation properties of 
the wavelets. It requires that the $V_{j}$ spaces contain, in a certain sense, all the polynomials of degree less or equal to $2 m-1$ in each variable.

The presentation of the second example requires more details. Let us consider the boundary value problem $D^{\star} a D u=f$ on $I=[0,1]$ with the Dirichlet conditions $u(0)=u(1)=0$ and $f \in L^{2}$, where

- $D=-i \frac{d}{d x}$ on the domain $V=H_{0}^{1}(0,1)=\left\{f \in H^{1}(0,1): f(0)=f(1)=0\right\}$;

- $D^{\star}=-i \frac{d}{d x}$ on the domain $V^{\star}=H^{1}(0,1)$

- $a$ is the operator of pointwise multiplication by the complex-valued function $a(x)$ which is bounded and accretive, i.e.,

$$
\begin{gathered}
|a(x)| \leq M=\|a\|_{\infty}, \\
\operatorname{Re} a(x) \geq 1 .
\end{gathered}
$$

Notice that no assumption of regularity is made for $a(x)$.

In [4], Tchamitchian and Auscher construct a Riesz basis $\left\{\tau_{\lambda}(x), \lambda \in \Lambda\right\}$ of $L^{2}(0,1), V=H_{0}^{1}(0,1)$ and the domain of the operator $T=D^{\star} a D, \mathcal{D}(T)$. The starting point is the construction of a wavelet basis, $\left(\theta_{\lambda}\right)_{\lambda \in \Lambda}$, of $L^{2}(0,1)$ which has the following specific cancellation property:

$$
\int_{0}^{1} \theta_{\lambda}(x) \frac{d x}{a(x)}=0
$$

Let us show how we proceed once these special wavelets are known: $\tau_{\lambda}$ is defined by

$$
\tau_{\lambda}(0)=0, \quad D \tau_{\lambda}(x)=2^{j} \frac{1}{a(x)} \theta_{\lambda}(x) .
$$

(Notice that $\tau_{\lambda} \in V$ when (1) holds.) Then we have

$$
\left(\tau_{\lambda}\right)_{\lambda \in \Lambda} \text { basis of } \mathcal{D}(T) \Longleftrightarrow\left(\theta_{\lambda}\right)_{\lambda \in \Lambda} \text { basis of } H^{1}(0,1)\left(=V^{\star}\right) \text {. }
$$

Since $\theta_{\lambda} \in V^{\star}$, we may then define the functions $\sigma_{\lambda}$ by

$$
2^{j} \sigma_{\lambda}(x)=D^{\star} \theta_{\lambda}(x)
$$

and finally we have

$$
D^{\star} a D \tau_{\lambda}(x)=4^{j} \sigma_{\lambda}(x)
$$

So, when $\tau_{\lambda}$ and $\sigma_{\lambda}$ have similar properties of size, decay and regularity, (4) can be seen as a "almost diagonalisation" of $T$. This is indeed the main motivation of the use of wavelets to characterise the domain of differential operators for which they constitute pseudo eigenfunctions. 
The collection of functions $\left(\tau_{\lambda}(x)\right)_{\lambda \in \Lambda}$ is a Riesz basis of $\mathcal{D}(T)$. The solution $u$ of the boundary value problem above introduced belongs to $\mathcal{D}(T)$. Thus, we may represent $u(x)$ by $u(x)=\sum_{\lambda \in \Lambda} \alpha_{\lambda} 4^{-j} \tau_{\lambda}(x)$ as soon as $f$ is represented by the sequence $\left(\alpha_{\lambda}\right)_{\lambda \in \Lambda}$ in the basis of $L^{2}(0,1)$ consisting of the functions $\sigma_{\lambda}(x), \lambda \in \Lambda$, i.e., as soon as $f(x)=\sum_{\lambda \in \Lambda} \alpha_{\lambda} \sigma_{\lambda}(x)$. In other words, one has decomposed the Green kernel of $D^{\star} a D$ with Dirichlet boundary conditions in a series $\sum_{\lambda \in \Lambda} 4^{-j} \tau_{\lambda}(x) \sigma_{\lambda}(y)$. Rigorously, one must complete the sets $\left(\tau_{\lambda}\right)_{\lambda \in \Lambda}$ and $\left(\sigma_{\lambda}\right)_{\lambda \in \Lambda}$ with two suitable bases of $V_{j_{0}}$ in order to obtain Riesz bases of $L^{2}(0,1)$. For simplicity, we skip this part of the problem and refer to [4] for the exact formulation and results. We shall concentrate on the construction of the set of functions $\left(\theta_{\lambda}\right)_{\lambda \in \Lambda}$ satisfying (1), which yields a Riesz basis of $L^{2}(0,1)$ and $V^{\star}$ (when completed with a suitable basis of $V_{j 0}$ ).

The third and last example concerns the construction of wavelets associated with a particular family of irregular meshes called $\Gamma$. These are defined as follows. We set $j_{0}=0$. Let $j_{p}$ be a strictly positive integer. Then we have $\Gamma:=\int_{j \geq 0}^{j_{p}} \Gamma_{j}$, where the $\Gamma_{j}$ are nested sequences of points satisfying the three following conditions:

1) $\Gamma_{0}:=\mathbb{Z}$ and $\Gamma_{j} \subset 2^{-j} \mathbb{Z}$

2) if $\Lambda_{j}:=\Gamma_{j+1} \backslash \Gamma_{j}$ then $\Lambda_{j} \subset 2^{-j-1} \mathbb{Z} \backslash 2^{-j} \mathbb{Z}$;

3) there exists $\rho \in \mathbb{N}^{\star}$ such that, if $\gamma=(2 k+1) 2^{-j-1} \in \Lambda_{j}$ then all points $\ell 2^{-j}$ with $|\ell-k| \leq \rho$, belong to $\Gamma_{j}$. This condition is called the cone condition.

One sees that the meshes $\Gamma_{j+1}$ are constructed from $\Gamma_{j}$ by addition of some points of $2^{-j-1} \mathbb{Z}$. Thus, $\Gamma_{j_{p}}$ contains pieces of $2^{-j_{p}} \mathbb{Z} \backslash 2^{-j_{p}+1} \mathbb{Z}$ nested in pieces of $2^{-j} \mathbb{Z}$ for $0<j<j_{p}$ and all of $\mathbb{Z}$. The cone condition ensures that the transition between a zone where $\Gamma_{j}$ is a coarse mesh and one where $\Gamma_{j}$ is refined is not too abrupt. Finally, when we represent the sets of points $\Gamma_{0}$ and $\Lambda_{j}, 0 \leq j \leq j_{p}$, in the position-scale half plane, we see that they form cones which cluster in the intervals of finest meshsize. To understand the choice of these meshes, we first recall how a wavelet series converges in $L^{2}(\mathbb{R})$.

Let us consider $f \in L^{2}(\mathbb{R})$ represented by the wavelet series

$$
f(x)=\sum_{k \in \mathbb{Z}} c_{0 k} g_{j_{0} k}(x)+\sum_{j \geq 0, k \in \mathbb{Z}} d_{j k} \psi_{j k}(x) .
$$

Let us assume that $f$ belongs locally to a Sobolev space $H^{s}, s>0$, for instance, on an open interval $I$. Then the wavelet coefficients of $f$ will satisfy:

$$
\sum_{k \in \mathbb{Z}}\left|c_{0 k}\right|^{2}+\sum_{j \geq 0, k \in \mathbb{Z}}\left|d_{j k}\right|^{2}<\infty
$$


and

$$
\sum_{j \geq 0, k \in \mathbb{Z}: k 2^{-j} \in I} 4^{j s}\left|d_{j k}\right|^{2}<\infty .
$$

The expression (6) is the characterisation of $L^{2}$, whereas (7) expresses the fact that $f$ is more regular on the interval $I$ (once more, we refer to chapter VI in [11] for the characterisation of functional spaces by wavelets). More generally, one knows that the more regular the function $f$ is in $x_{0}$, the quicker the coefficients $d_{j k}$ decrease when $j \rightarrow+\infty$ and $k 2^{-j} \rightarrow x_{0}$ (see [6] and [7] for complete and precise results). Thus, when $f$ is regular on the larger part of the domain under consideration and singular (or pseudo-singular) on the remaining part, the series (5) will be sparse and, in the scale-position half plane, the nonnegligible coefficients will be localised in cones condensing near the singularities.

Let us consider such kind of functions and assume that $f$ is well described by its sampling points on the above defined mesh $\Gamma$. The wavelets which are constructed in [13] are associated with the points of $\Lambda_{j}$, i.e., they allow to represent $f$ intrinsically by its wavelet coefficients computed from the sampled values on $\Gamma$.

In the following sections, we shall refer to the above cases as respectively E1,E2, and E3.

\section{$\S 3$ Multiresolution analyses}

The definition of the multiresolution analysis associated with any of the three above described problems is divided in two steps: the geometrical and the functional properties. In the first step, we define the sets of dyadic points which will approximate the geometry of the situation, i.e., the sets $\Gamma_{j}$. The definition of the set of wavelet labels, $\Lambda$, will follow. In the second step, we define the functional spaces $V_{j}$ associated with these meshes.

\subsection{Geometrical aspects}

We already gave a description of the construction of the meshes $\Gamma_{j}$ (and $\Lambda_{j}$ ) for E3. Let us now do it for E1 and E2. In both these cases it consists of restricting the dyadic grids $2^{-j} \mathbb{Z}$.

E1- $\Gamma_{j} \subset 2^{-j} \mathbb{Z}^{n}$ is the set of all $\gamma=2^{-j} k$ whose distance to $\partial \Omega$ is greater or equal to $(m+1) 2^{-j}$.

E2- For $j \geq 0, \Gamma_{j}$ is defined by: $\Gamma_{j}=\left\{k 2^{-j}: 0 \leq k \leq 2^{j}\right\}$.

Thus, in all cases, the so defined meshes satisfy the following properties:

- $\Gamma_{j} \subset \Gamma_{j+1}$ for $j, j_{0} \leq j \leq j_{p}$,

- $\int_{j=j_{0}}^{j_{p}} \Gamma_{j}$ is dense in some domain $\Omega_{p}$ 
and the set of the wavelet labels $\Lambda$ is given by:

$$
\Lambda=\bigcup_{j=j_{0}}^{j_{p}} \Lambda_{j} \text { with } \Lambda_{j}=\Gamma_{j+1} \backslash \Gamma_{j}
$$

where, for E3: $j_{0}=0$ and $j_{p}$ is a strictly positive integer given by the mesh $\Gamma, \Omega_{p}$ is $\Gamma$; for $\mathrm{E} 2: j_{0}=0$ and $j_{p}=+\infty, \Omega_{p}$ is the interval [0,1]; for E1: $\Omega_{p}$ is the open set $\Omega, j_{p}=+\infty$ and $j_{0}$ is an integer when $\Omega$ is bounded.

\subsection{Functional properties}

The main idea is to construct the $V_{j}$ spaces in such a way that for any function $f \in V_{j}$, the $\ell^{2}$-norm of its values on $\Gamma_{j},(f(\gamma))_{\gamma \in \Gamma_{j}}$, is equivalent to the $L^{2}$-norm of $f$. This means that there should exist two positive constants, $A$ and $B$, independent of $j$, such that

$$
A \int|f|^{2} \leq \sum_{\gamma \in \Gamma_{j}} c_{j}(\gamma)|f(\gamma)|^{2} \leq B \int|f|^{2},
$$

where $c_{j}(\gamma)$ is a normalisation constant equal to: $2^{-\frac{j}{2}}$ for E2, $2^{-\frac{j n}{2}}$ for E1 and the distance to the nearest neighbour of $\gamma$ in $\Gamma_{j}$ for E3. In other words, we need to prove:

Propostion. On $V_{j}$, the norm defined by the scalar product

$$
(f, g)_{j}=\sum_{\gamma \in \Gamma_{j}} c_{j}(\gamma) f(\gamma) \overline{g(\gamma)}
$$

is equivalent to the $L^{2}$ norm, uniformly with respect to $j$.

This result is nothing but a theorem of sampling on $\Gamma_{j}$. It is obtained by the construction of the continuous functions $\Delta_{j, \gamma} \in V_{j}$, which have the following property: for any $\gamma, \gamma^{\prime} \in \Gamma_{j}, \Delta_{j, \gamma}\left(\gamma^{\prime}\right)=1$ if $\gamma=\gamma^{\prime}$ and $\Delta_{j, \gamma}\left(\gamma^{\prime}\right)=0$ if $\gamma \neq \gamma^{\prime}$ (the so called Lagrangian property). The fact that the set $\left(\Delta_{j, \gamma}(x)\right)_{\gamma \in \Gamma_{j}}$ forms an unconditional basis of $V_{j}$ is equivalent to the proposition. The motivation of this idea will become clear in the next section. Then, we shall see that it allows us to define the required supplementary set of $V_{j}$ in $V_{j+1}$ very naturally.

Before we give the description of the multiresolution analysis in each particular case, let us mention which properties of the classical multiresolution analysis of $L^{2}(\mathbb{R})$ are preserved. Since the functions of $V_{j}$ are completely defined by their values on the meshes $\Gamma_{j}$, the features of the new multiresolution analysis come very naturally from its geometrical properties as described above. We have, with the same notations as before, 
(1) $V_{j} \subset V_{j+1}$,

(2) $\bigcup_{j=j_{0}}^{j_{p}} V_{j}$ is dense in $L^{2}\left(\Omega_{p}\right)$

(3) for each $V_{j}$, there exists a family of regular and well localised functions, $\left(\Delta_{j, \gamma}(x)\right)_{\gamma \in \Gamma_{j}}$, which constitutes a Riesz basis of $V_{j}$.

Neither the invariance under dyadic translation nor the scaling properties are preserved. Notice that for this reason, the bases of the $V_{j}$ spaces are no longer deduced by scaling from the basis of $V_{0}$. Moreover, the basis of each $V_{j}$ is not formed by a set of dilated and translated versions of a single function. However, their localisation is ensured by the following estimates, so-called standard estimates: for $|\alpha| \leq r$ and two constants $C$ and $\zeta$ (depending only on $n$ and $r$ )

$$
\left|\partial^{\alpha} \Delta_{j, \gamma}(x)\right| \leq C 2^{j|\alpha|} 2^{n \frac{j}{2}} \exp \left[-\zeta 2^{j}|x-\gamma|\right] .
$$

Remark. Point (2) deserves some additional attention for E3. We shall return to this in the remarks at the end of this section.

E1- Let $Q(j, k)$ be the dyadic cubes defined by $2^{j} x-k \in[0,1]^{n}, j \in \mathbb{Z}, k \in \mathbb{Z}^{n}$. Let $V_{j}$ be the space of compactly supported functions of regularity $C^{2 m-2}$, with a support included in $\Omega$ and at a distance to $\partial \Omega$ of at least $2^{-j}$, and of which the restriction to $Q(j, k)$ coincides with a polynomial of degree less or equal to $2 m-1$ in each variable. We introduce the basic spline, $s_{m}$, by the convolution of the characteristic function on the unit cube: $s_{m}(x-m)=\chi \star \cdots \star \chi(2 m$ times). Then the set $\left\{s_{j, \gamma}(x), \gamma \in \Gamma_{j}\right\}$, defined by $s_{j, \gamma}(x)=2^{n \frac{j}{2}} s_{m}\left(2^{j} x-k\right)$, where $\gamma=k 2^{-j} \in \Gamma_{j}$, is a Riesz basis of $V_{j}$. From this basis one constructs the desired Lagrangian basis by application of a transfer matrix which preserves estimates of type 1.1(iii).

E2- $V_{j}, j \geq 0$, is the space of square integrable piecewise linear splines $f(x)$ of the form

$$
f(x)=\sum_{\gamma \in \Gamma_{j}} 2^{-\frac{j}{2}} f(\gamma) \Delta_{j, \gamma}(x), \quad x \in[0,1] .
$$

The so called hat-function $\Delta(x)$ is defined by $\Delta(x)=\sup \{1-|x|, 0\}, x \in \mathbb{R}$. The $\Delta_{j, \gamma}(x), \gamma \in \Gamma_{j}$ are the restrictions to $[0,1]$ of the functions $\Delta_{j, k}(x)=$ $2^{\frac{j}{2}} \Delta\left(2^{j} x-k\right)$ with $k \in 2^{j} \Gamma_{j}$.

E3- $V_{j}$ consists of the continuous functions of $L^{2}(\mathbb{R})$ which are completely determined by their values on $\Gamma_{j}$ according to the following procedure. The first part of the procedure consists of defining the values of $f$ on the whole set $2^{-j} \mathbb{Z}$ by "completing" $\frac{\mathbb{Z}}{2}$, then $\frac{\mathbb{Z}}{4}$ and so until $2^{-j} \mathbb{Z}$. Let us consider $\frac{k}{2} \in \frac{\mathbb{Z}}{2}$. If $\frac{k}{2} \in \Gamma_{j}, f\left(\frac{k}{2}\right)$ is given. If $\frac{k}{2} \notin \Gamma_{j}$, then $f\left(\frac{k}{2}\right)$ is defined by dyadic interpolation from its values on $\mathbb{Z}$ ( $\mathbb{Z}$ always belongs to $\Gamma_{j}$ by definition). We continue until $f\left(k 2^{-j}\right)$ is defined for all $k \in \mathbb{Z}$. Then, in the second part of the procedure, 
$f$ is defined on the whole real line by successive dyadic interpolation from its values on $2^{-j} \mathbb{Z}$. Of course, $\Delta$ is the fundamental interpolant of the process of dyadic interpolation. The functions $\Delta_{j, \gamma}(x)$ are compactly supported and, due to the cone condition, their support is uniformly controlled by $c_{j}(\gamma)$, i.e., by the local density of the irregular mesh $\Gamma_{j}$.

Remarks. Our first remark concerns the treatment of the boundary conditions in E2. As we already said, $V_{j}$ must be in $V^{\star}=H^{1}(0,1)$. This implies that the functions of $V_{j}$ must not satisfy any condition at the boundary of the interval. This is ensured by keeping the end points of the interval, 0 and 1 , in $\Gamma_{j}$ : the boundary values of $\Gamma_{j}$ imply boundary conditions on the functions of $V_{j}$. According to this rule, Auscher and Tchamitchian propose all the possible boundary conditions by choosing a suitable definition of $\Gamma_{j}$ :

$$
\Gamma_{j}=\left\{k 2^{-j}: \operatorname{dist}\left(k 2^{-j}, \partial I\right) \geq 2^{-j}\right\} \cup B V, \forall j \geq 0,
$$

where $B V$ denotes the set of boundary points in $\Gamma_{j}$ adapted to each different situation. In our example, $B V=\{0,1\}$ whereas the example where $V=H^{1}(0,1)$ and $V^{\star}=H_{0}^{1}(0,1), B V$ is the empty set. The latter ensures that the functions of $V_{j}$ are equal to 0 at the end points of the interval.

Our second remark, still about E2, concerns the choice of the spaces $V_{j}$. The spaces of piecewise linear splines are the simplest one can imagine. The authors of [4] chose them for this reason. It appears that the piecewise linear splines are sufficient for their purpose, which is not to construct wavelets adapted to the differential operator $T$ for their own sake but to use them to prove Kato's conjecture on open sets of $\mathbb{R}$. As usual, the very low regularity of the multiresolution analysis is the price to pay for the simplicity of the piecewise linear splines. There is however no reason to think that the construction could not hold for more complicated (and more regular) spaces $V_{j}$.

In contrast to the above, the regularity of the multiresolution analysis constructed for E1 is essential, because the wavelet basis which is developed from it is used for the characterisation of the Hölder spaces. Let us remark that only the splines of odd order are considered. Actually, it is known (see [11], p.24-25) that the splines of even order cannot lead to cardinal (or Lagrangian) splines.

Finally, we return to point (2) for case E3. The functional space $\underset{j}{\cup V_{j}}$ is not a classical space. For convenience, we denoted it by $L^{2}(\Gamma)$ without any ambition of a precise description. For the present time, $L^{2}(\Gamma)$ has no other definition than this one which is derived from the Lagrangian multiresolution analysis.

\section{$\S 4$ Construction of the wavelets}

Now the multiresolution analysis has been defined, the construction of wavelets may start. We have divided it in two parts. The first part is devoted to the construction 
of the wavelets which are called "classical" because they are obtained by considering the usual orthogonal supplement, $W_{j}$, of $V_{j}$ in $V_{j+1}$. This will lead to the desired orthonormal bases of $W_{j}$ for E1 and E2 and the not less desired Riesz basis of $W_{j}$ for E3.

In the second stage, we describe the construction of the "non-classical" wavelets, which are called so because they are obtained by considering oblique supplements of $V_{j}$ in $V_{j+1}$. We shall explain how the wavelets $\theta_{\lambda}(x), \lambda \in \Lambda_{j}$, which satisfy the special cancellation property (2.1), are constructed and we shall propose an alternative for the Riesz basis defined for E3 earlier.

\subsection{The "classical" wavelets}

Let us consider the space $W_{j}$, the orthogonal supplement of $V_{j}$ in $V_{j+1}$ with respect to the $L^{2}$-scalar product, i.e.,

$$
W_{j}:=\left\{f \in V_{j+1}:\langle f, v\rangle=0 \quad \forall v \in V_{j}\right\} .
$$

As we have already pointed it out, we do not have direct access to the spaces $W_{j}$. However, we have defined the spaces $V_{j}$ of the multiresolution analysis in such a way that we perfectly know how to construct an other supplement of $V_{j}$ in $V_{j+1}$, which we call $U_{j}$. Let us now give the basic recipe for their construction. Since, from the theorem of sampling on $\Gamma_{j+1}$, the functions of $V_{j+1}$ are completely determined by their values on $\Gamma_{j+1}$, and since $\Gamma_{j+1}$ is the disjoint union of $\Gamma_{j}$ and $\Lambda_{j}$, we can define $U_{j}$ as a closed subspace of $V_{j+1}$ consisting of the functions that are completely determined by their values on $\Lambda_{j}$. Let us call $\left(u_{\lambda}(x)\right)_{\lambda \in \Lambda_{j}}$ a Riesz basis of $U_{j}$, then we have

$$
\forall f \in U_{j} \quad f(x)=\sum_{\lambda \in \Lambda_{j}} f(\lambda) u_{\lambda}(x) .
$$

This ensures that $V_{j+1}$ is the direct sum of $V_{j}$ and $U_{j}$. Later we shall see the precise definition of the two different $U_{j}$, constructed for $\mathrm{E} 1$ and $\mathrm{E} 2$ on the one hand and for E3 on the other hand. From this definition, we shall see that we know $\left(u_{\lambda}(x)\right)_{\lambda \in \Lambda_{j}}$ explicitly.

Now we have at our disposal the three spaces, $V_{j+1}, V_{j}$, and $U_{j}$, such that

$$
V_{j+1}=V_{j} \oplus U_{j},
$$

where $\oplus$ denotes the direct sum. Moreover, we know a Riesz basis for the different parts:

- $\left(u_{\lambda}(x)\right)_{\lambda \in \Lambda_{j}}$ is a Riesz basis of $U_{j}$;

- $\left(\Delta_{j, \gamma}(x)\right)_{\lambda \in \Gamma_{j}}$ is a Riesz basis of $V_{j}$. 
We aim at the construction of a Riesz basis for $W_{j}$, the orthogonal supplement of $V_{j}$ in $V_{j+1}$. Therefore, we introduce $\Pi_{j}$, the orthogonal projection from $V_{j+1}$ onto $V_{j}$; then $I-\Pi_{j}$ is the orthogonal projection from $V_{j+1}$ onto $W_{j}$, and it is an isomorphism between $U_{j}$ and $W_{j}$. Further, it follows that

The family of functions, $\left(w_{\lambda}(x)\right)_{\lambda \in \Lambda_{j}}$, defined by

$$
w_{\lambda}=\left(I-\Pi_{j}\right) u_{\lambda} \quad \forall \lambda \in \Lambda_{j}
$$

forms a Riesz basis of $W_{j}$. All $w_{\lambda}(x)$ satisfy standard estimates (3.1).

Let us now return to the definition of $U_{j}$.

E1,

$\mathrm{E} 2-$ For $\mathrm{E} 1$, as well as for $\mathrm{E} 2, U_{j}$ is the space of functions of $V_{j+1}$ which are equal to 0 on $\Gamma_{j}$. The collection of functions $\left(u_{\lambda}(x)\right)_{\lambda \in \Lambda_{j}}$ where $u_{\lambda}(x)=\Delta_{j+1,2 k+1}(x)$, $\lambda=(2 k+1) 2^{-j-1} \in \Lambda_{j}$, forms a Riesz basis of $U_{j}$.

E3- For E3, $U_{j}$ is the supplement of $V_{j}$ in $V_{j+1}$, oblique with respect to the $L^{2}$-scalar product but orthogonal with respect to the scalar product on $V_{j+1},(,)_{j+1}$. Together with the fact that the functions of $U_{j}$ are completely determined by their values on $\Lambda_{j}$, this definition allows to construct a Riesz basis of $U_{j}$ : for $\lambda \in \Lambda_{j}, u_{\lambda}(x)$ is defined by

$$
\left\{\begin{array}{l}
u_{\lambda} \in V_{j+1} ; \\
u_{\lambda}\left(\lambda^{\prime}\right)=\delta_{\lambda, \lambda^{\prime}}, \quad \text { when } \lambda^{\prime} \in \Lambda_{j} ; \\
u_{\lambda}(\gamma)=-\frac{c_{j+1}(\lambda)}{c_{j+1}(\gamma)} \overline{\Delta_{j, \gamma}(\lambda)}, \quad \text { when } \gamma \in \Gamma_{j} .
\end{array}\right.
$$

Then, it is easy to show that the functions $\left(u_{\lambda}(x)\right)_{\lambda \in \Lambda_{j}}$ form a Riesz basis of $U_{j}$.

We see that the space $U_{j}$, so-defined for this example, is fundamentally different from the previous one used for E1 and E2. Here $U_{j}$ is a "wavelet space" since it is orthogonal to $V_{j}$ with respect to a scalar product which is equivalent, on $V_{j+1}$, to the $L^{2}$-scalar product. We shall return to this in the next section.

Before this, however, we give few comments about the projection $\Pi_{j}$. This projection is explicitly known but its expression deserves a little attention.

E1,

E2- For E1 and E2, one obtains the explicit expression of $\Pi_{j}$ by orthonormalisation the Riesz basis $\left(\Delta_{j, \gamma}\right)_{\gamma \in \Gamma_{j}}$ of $V_{j}$. The orthonormal basis of $V_{j}$ is denoted $\left(v_{\gamma}(x)\right)_{\gamma \in \Gamma_{j}}$ and, hence, the projection $\Pi_{j}$ is given by

$$
\forall f \in V_{j+1} \quad\left(\Pi_{j} f\right)(x)=\sum_{\gamma \in \Gamma_{j}}\left\langle f, v_{\gamma}\right\rangle v_{\gamma}(x),
$$


which gives for $\left(w_{\lambda}(x)\right)_{\lambda \in \Lambda_{j}}$ :

$$
w_{\lambda}(x)=u_{\lambda}(x)-\sum_{\gamma \in \Gamma_{j}}\left\langle u_{\lambda}, v_{\gamma}\right\rangle v_{\gamma}(x)
$$

Then, by orthonormalisation of $\left(w_{\lambda}(x)\right)_{\lambda \in \Lambda_{j}}$, we obtain the orthonormal basis $\left(\psi_{\lambda}(x)\right)_{\lambda \in \Lambda_{j}}$ of $W_{j}$.

E3- For E3, one shows that the biorthogonal family $\left(\tilde{\Delta}_{j, \gamma}\right)_{\gamma \in \Gamma_{j}}$ of $\left(\Delta_{j, \gamma}\right)_{\gamma \in \Gamma_{j}}$ forms also a Riesz basis of $V_{j}$. Thus, $\Pi_{j}$ is written in this case as:

$$
\forall f \in V_{j+1} \quad\left(\Pi_{j} f\right)(x)=\sum_{\gamma \in \Gamma_{j}}\left\langle f, \tilde{\Delta}_{j, \gamma}\right\rangle \Delta_{j, \gamma}(x),
$$

or equivalently by,

$$
\left(\Pi_{j} f\right)(x)=\sum_{\gamma \in \Gamma_{j}}\left\langle f, \Delta_{j, \gamma}\right\rangle \tilde{\Delta}_{j, \gamma}(x)
$$

which gives for $\left(w_{\lambda}(x)\right)_{\lambda \in \Lambda_{j}}$ :

$$
w_{\lambda}(x)=u_{\lambda}(x)-\sum_{\gamma \in \Gamma_{j}}\left\langle u_{\lambda}, \Delta_{j, \gamma}\right\rangle \tilde{\Delta}_{j, \gamma}(x) .
$$

The family $\left(w_{\lambda}(x)\right)_{\lambda \in \Lambda_{j}}$ as well as its biorthogonal system $\left(\tilde{w}_{\lambda}(x)\right)_{\lambda \in \Lambda_{j}}$ is a Riesz basis of $W_{j}$.

Thus, we have constructed an orthonormal basis of $W_{j}$ for E1 and E2 consisting of the corresponding orthonormalised $\left(\psi_{\lambda}(x)\right)_{\lambda \in \Lambda_{j}}$ and a system of biorthogonal bases of $W_{j}$ for E3, consisting of the collections of functions $\left(w_{\lambda}(x)\right)_{\lambda \in \Lambda_{j}}$ and $\left(\tilde{w}_{\lambda}(x)\right)_{\lambda \in \Lambda_{j}}$.

\subsection{The "non-classical" wavelets}

The non-classical wavelets are obtained by considering the proper oblique supplements of $V_{j}$ in $V_{j+1}$. These supplements are not orthogonal to $V_{j}$ with respect to the $L^{2}$-scalar product, but they are orthogonal with respect to another suitable scalar product (E3) or even, with respect to a bilinear (E2) or sesquilinear form (see another example of boundary conditions in [4]). Depending on the choice of scalar product, or bi- (sesqui-) linear form, this specific orthogonality may imply, for the wavelets, specific cancellation properties. It may also allow to construct 
wavelets suitable for practical use in the sense that the associated analysis and synthesis algorithms are simple and fast. The two families of wavelets that we want to construct illustrate both possibilities.

We have already seen an example of construction of these "non-classical" wavelets for E3. They were obtained by considering the space $U_{j}$, supplement of $V_{j}$ in $V_{j+1}$, nonorthogonal with respect with the $L^{2}$-scalar product but orthogonal with respect to the scalar product on $V_{j+1},(,)_{j+1}$, which is equivalent, on $V_{j+1}$ to the $L^{2}$-scalar product, i.e.,

$$
U_{j}:=\left\{f \in V_{j+1}:(f, v)_{j+1}=0 \quad \forall v \in V_{j}\right\} .
$$

The set of functions $\left(u_{\lambda}\right)_{\lambda \in \Lambda_{j}}$ defined by (2) forms a Riesz basis of $U_{j}$, of which the elements are mutually orthogonal for the scalar product $(,)_{j+1}$. The very simple definition of the functions $u_{\lambda}(x), \lambda \in \Lambda_{j}$, and the simplicity of the associated analysis and synthesis algorithms (see [13]) make this basis a good candidate for practical applications.

To construct the wavelets $\left(\theta_{\lambda}(x)\right)_{\lambda \in \Lambda_{j}}$ of the example E2, we define the space $X_{j}$, supplement of $V_{j}$ in $V_{j+1}$, nonorthogonal with respect to the $L^{2}$-scalar product but orthogonal with respect to the bilinear form $B($,$) , symmetric and continuous$ on $L^{2}(0,1)$ :

$$
B(f, g)=\int_{0}^{1} f(x) \frac{1}{a(x)} g(x) d x .
$$

Thus $X_{j}$ is defined by

$$
X_{j}:=\left\{f \in V_{j+1}: B(f, v)=0 \quad \forall v \in V_{j}\right\},
$$

and we have

$$
V_{j+1}=V_{j} \oplus X_{j},
$$

where $\oplus$ denotes the direct sum. Following the same approach as before, we define $P_{j}$, the projection associated with this direct sum from $V_{j+1}$ onto $V_{j}$, the null-space of which is $X_{j}$. Then $I-P_{j}$ is the projection from $V_{j+1}$ onto $X_{j}$ which is an isomorphism from $W_{j}$ to $X_{j}$, and functions defined by

$$
\chi_{\lambda}(x)=\psi_{\lambda}(x)-P_{j}\left(\psi_{\lambda}\right)(x), \quad \forall \lambda \in \Lambda_{j}
$$

form a Riesz basis of $X_{j}$. The functions $\theta_{\lambda}(x)$ are obtained by orthonormalisation with respect to the form $B$, as follows. Let $M$ be the matrix of coefficients $B\left(\chi_{\lambda}, \chi_{\lambda^{\prime}}\right), M$ is bounded and accretive on $\ell^{2}\left(\Lambda_{j}\right)$. Let $\beta\left(\lambda, \lambda^{\prime}\right)$ be the coefficients of the matrix $M^{-\frac{1}{2}}$. From the estimates of $\psi_{\lambda}(x)$, it is shown in [4] that these coefficients satisfy the following estimates for $\lambda^{\prime}=\left(\ell+\frac{1}{2}\right) 2^{-j}$ and $\lambda=\left(k+\frac{1}{2}\right) 2^{-j} \in \Lambda_{j}$ :

$$
\mid \beta\left(\lambda, \lambda^{\prime}\right) \leq C \exp [-\zeta|k-\ell|] .
$$


Then the functions defined by

$$
\theta_{\lambda}(x)=\sum_{\lambda^{\prime} \in \Lambda_{j}} \beta\left(\lambda, \lambda^{\prime}\right) \chi_{\lambda^{\prime}}(x), \quad \forall \lambda \in \Lambda_{j}
$$

form a Riesz basis of $X_{j}$, orthogonal for $B$. We shall say that $\left(\theta_{\lambda}(x)\right)_{\lambda \in \Lambda_{j}}$ is a $B$-orthogonal Riesz basis of $X_{j}$.

Notice that we can describe the projection $P_{j}$ by the same procedure applied to the Riesz basis of $V_{j}$. If we denote the $B$-orthogonal Riesz basis of $V_{j}$ by $\left(v_{\gamma}^{\star}(x)\right)_{\lambda \in \Gamma_{j}}$, we have

$$
\left(P_{j} f\right)(x)=\sum_{\gamma \in \Gamma_{j}} B\left(f, v_{\gamma}^{\star}\right) v_{\gamma}^{\star}(x), \quad \forall f \in V_{j+1} .
$$

It is easy to see that the spaces $V_{j}$ of the multiresolution analysis of $L^{2}(0,1)$ defined for $\mathrm{E} 2$, are generated by the monomials 1 and $x$. Thus, the $B$-orthogonality of the spaces $X_{j}$ and $V_{j}$ implies the desired cancellation property for the wavelets $\theta_{\lambda}(x), \lambda \in \Lambda_{j}$, that form a basis of $X_{j}$. We have

$$
\int_{0}^{1} \frac{1}{a(x)} \theta_{\lambda}(x) d x=\int_{0}^{1} x \frac{1}{a(x)} \theta_{\lambda}(x)=0 .
$$

Notice that $\theta_{\lambda}(x) \in V^{\star}$. Effectively, since the points 0 and 1 belong to $\Gamma_{j}$ for all $j \geq 0$, and since $\left(\theta_{\lambda}(x)\right)_{\lambda \in \Lambda_{j}} \in V_{j+1}$, from the first remark in Section 3, it follows that $\left(\theta_{\lambda}(x)\right)_{\lambda \in \Lambda_{j}} \in V^{\star}$.

Remark. Notice that E2 combines two distinct goals. The first goal is the construction of a basis of $L^{2}(0,1)$, consisting of wavelets which show precise boundary values. This is achieved by the definition of the multiresolution analysis. More precisely, once the idea of Lagrangian multiresolution analysis is adopted, the problem is reduced to the suitable choice of the geometrical aspects of the multiresolution analysis. The second goal consists of constructing wavelets adapted to a differential operator. It is achieved by the right choice of the supplement of $V_{j}$ in $V_{j+1}$.

This part is really separable from the first one. Indeed the construction of such wavelets was performed in [3], independently of any interest for the interval. In the same way the construction of wavelets on an interval which show conditions at the boundary of the interval can be found in [1].

\section{$\S 5$ The proof that the wavelets form a basis of $\bar{j} \overline{V_{j}}$}

The bases of the spaces $W_{j}$ form a basis of $\bar{j} \overline{V_{j}}$ since the $W_{j}$ are mutually orthogonal and since their union is dense in $\bar{j} V_{j}$ by construction. Hence, the family of wavelets 
$\left(\psi_{\lambda}(x)\right)_{\lambda \in \Lambda}$ defined for E1 (E2) forms an orthonormal basis of $L^{2}(\Omega)\left(L^{2}(0,1)\right)$. The functions $\left(w_{\lambda}(x)\right)_{\lambda \in \Lambda}$ and $\left(\tilde{w}_{\lambda}(x)\right)_{\lambda \in \Lambda}$ form a system of biorthogonal bases of $L^{2}(\Gamma)$. Thus, the problem addressed in E1 has been solved, as well as part of the problem described in E3.

In this paragraph, we shall present the strategy to determine whether the bases $\left(u_{\lambda}(x)\right)_{\lambda \in \Lambda}$ and $\left(\theta_{\lambda}(x)\right)_{\lambda \in \Lambda}$ form a basis of $L^{2}(\Gamma)$ and $L^{2}(0,1)$ respectively. We must warn the reader that the result for E3 is incomplete, i.e., whether the functions $\left(u_{\lambda}(x)\right)_{\lambda \in \Lambda}$ form a Riesz basis of $L^{2}(\Gamma)$ remains an open question. We shall comment on this at the end of this section. As we noticed in the introduction, this fact does not follow immediately from the construction of the wavelets, as it does for the classical wavelets, but it requires a specific proof. As before, we shall first present a general approach and later, we shall mention the particularities for each example. By $\theta_{\lambda}(x), \lambda \in \Lambda$, we denote the wavelets which are considered (in particular $\theta_{\lambda}(x)$ and $u_{\lambda}(x)$ constructed in the previous section). In the following we denote by $I$ an interval of $\mathbb{R}$, finite or infinite. The wavelets we consider satisfy the standard estimates:

$$
\left|\theta_{\lambda}^{(m)}(x)\right| \leq C 2^{m \frac{j}{2}} \exp \left\{-\zeta 2^{j}|x-\lambda|\right\}
$$

for all $x \in I$ and $\lambda \in \Lambda$ and for all $m$ such that $|m| \leq r$, where $r$ is the regularity of the multiresolution analysis from which the wavelets are derived $(C$ and $\zeta$ are two positive constants). Moreover, the wavelets satisfy one of the following cancellation properties:

$$
\begin{aligned}
& \int_{I} \theta_{\lambda}(x) d x \text { vanishes } \\
& \text { or } \\
& \int_{I} \theta_{\lambda}(x) d x \text { is small in a sense which will be made precise later. }
\end{aligned}
$$

Thus, we need to prove that two strictly positive constants $A$ and $B$ exist such that for any sequence $\left(\alpha_{\lambda}\right)$ we have

$$
A \sum_{\lambda \in \Lambda}\left|\alpha_{\lambda}\right|^{2} \leq\left\|\sum_{\lambda \in \Lambda} \alpha_{\lambda} \theta_{\lambda}\right\|^{2}
$$

and

$$
\left\|\sum_{\lambda \in \Lambda} \alpha_{\lambda} \theta_{\lambda}\right\|^{2} \leq B \sum_{\lambda \in \Lambda}\left|\alpha_{\lambda}\right|^{2}
$$

The two inequalities will be treated separately. We start with (4). 


\subsection{Inequality (4)}

The proof of (4) follows from the combination of the properties (1) and (2). We shall need the following concepts of vaguelettes and Carleson condition to formulate it.

Definition 1. Let $m_{\lambda}(x)$ be a sequence of functions defined on $I$. Then, $\left(m_{\lambda}\right)$ is said to be a collection of vaguelettes on $I$ if the functions $m_{\lambda}(x)$ satisfy the standard estimates (1) and the relation

$$
\int_{I} m_{\lambda}(x) d x=0
$$

It is clear that the classical wavelets of $L^{2}(\mathbb{R})$ form a family of vaguelettes on $\mathbb{R}$. But, where -on the one hand- the wavelets are translated and dilated versions of one single function (the mother wavelet), -on the other hand- by the standard estimates, the vaguelettes only have approximately the same shape as obtained from an initial pattern by dilations and translations. In the regular case where $I=\mathbb{R}$, it is known that (4) holds as soon as the functions $\theta_{\lambda}(x)$ defined on $I$ are a family of vaguelettes on $I$ [12]. For the case when $I$ is a finite interval of $\mathbb{R}$ it is shown in [4].

In fact, the condition (5) is somewhat too strong. To satisfy (4), it is sufficient that the numbers $\left(\int_{I} m_{\lambda}(x) d x\right), \lambda \in \Lambda$, are small, in a sense that is quantified by the Carleson condition. Let $I_{\mu}$ be the dyadic interval centered on $\mu=\left(k+\frac{1}{2}\right) 2^{-j} \in$ $\Lambda_{j}$,i.e., $I_{\mu}=\left[k 2^{-j},(k+1) 2^{-j}\right]$. We have the following definition.

Definition 2. A complex-valued sequence $\left(c_{\lambda}\right)$ is said to satisfy a Carleson condition if there exists a constant $C \geq 0$ such that for all $\lambda \in \Lambda$, one has

$$
\sum_{\mu \in \Lambda, I_{\mu} \subset I_{\lambda}}\left|c_{\mu}\right|^{2} \leq C\left|I_{\lambda}\right|
$$

We now have the result (see [4]):

Theorem 1. Let $\theta_{\lambda}, \lambda \in \Lambda$, be a sequence of functions which satisfies the standard estimates (1). Then, the following two assertions are equivalent:

$$
\begin{aligned}
& \text { (i) }\left\|\sum \alpha_{\lambda} \theta_{\lambda}\right\|^{2} \leq C \sum\left|\alpha_{\lambda}\right|^{2} ; \\
& \text { (ii) }\left(\int_{I} \theta_{\lambda}(x) d x\right) \quad \text { satisfies a Carleson condition. }
\end{aligned}
$$

E2- We give the main arguments used to prove that $\left(\int_{I} \theta_{\lambda}(x) d x\right)$ satisfies (ii). From the definition (4.4) of the functions $\theta_{\lambda}(x)$, we just have to notice that 
$\left(\int_{I} \chi_{\lambda}(x) d x\right)$ satisfies a Carleson condition (see the estimates (4.3) of the matrix elements). We recall that,

$$
\int_{I} \chi_{\lambda}(x) d x=\int_{I} \psi_{\lambda}(x) d x-\int_{I} \frac{1}{a(x)} q_{\lambda}(x)
$$

where $q_{\lambda}(x)=\psi_{\lambda}(x) \sum_{\gamma \in \Gamma_{j}}\left(\int_{I} v_{\gamma}^{\star}(x) d x\right) v_{\gamma}^{\star}(x)$. With the standard estimates of the functions $v_{\gamma}^{\star}(x)$ and the orthogonality of $V_{j}$ and $W_{j}$ (which implies that $\psi_{\lambda}(x), \lambda \in \Lambda_{j}$, is a family of vaguelettes on $\left.L^{2}(0,1)\right)$, we conclude that $q_{\lambda}(x)$, $\lambda \in \Lambda_{j}$ is a collection of vaguelettes on $L^{2}(0,1)$. This allows us to conclude (see Theorem 6, page 168 [4]) that $\left(\frac{1}{a} q_{\lambda}\right)$ satisfies a Carleson condition.

E3- The proof of (ii) starts by noticing that most of the wavelets $u_{\lambda}(x), \lambda \in \Lambda$ have zero mean value. Roughly speaking, the wavelets of which the support is included in the piece taken from $2^{-j-1} \mathbb{Z}$ of $\Gamma_{j+1}$ are not perturbed by the transition zones between two scales of $\Gamma_{j+1}$. Indeed, they are just the wavelets which would be obtained from a Lagrangian multiresolution analysis of $L^{2}(\mathbb{R})$. The last step of the proof consists of showing that the number of wavelets which do not satisfy the cancellation property (5) is sufficiently small.

\subsection{Inequality (3)}

The inequality (3) is obtained by duality, due to the following well known result. Lemma. Let $H$ be a separable Hilbert space and let $\left(e_{k}\right)$ and $\left(f_{k}\right)$ be two biorthonormal sequences of $H$, i.e., $\left\langle e_{k}, f_{\ell}\right\rangle=\delta_{k, l}$ for all $k$ and $\ell$. If for every sequence of complex numbers $\left(\alpha_{k}\right)$

$$
\begin{aligned}
& \left\|\sum \alpha_{k} e_{k}\right\|^{2} \leq C_{1} \sum\left|\alpha_{k}\right|^{2}, \\
& \left\|\sum \alpha_{k} f_{k}\right\|^{2} \leq C_{2} \sum\left|\alpha_{k}\right|^{2},
\end{aligned}
$$

then

$$
\begin{aligned}
& \sum\left|\alpha_{k}\right|^{2} \leq C_{2}\left\|\sum \alpha_{k} e_{k}\right\|^{2} \\
& \sum\left|\alpha_{k}\right|^{2} \leq C_{1}\left\|\sum \alpha_{k} f_{k}\right\|^{2} .
\end{aligned}
$$

E2- We apply this lemma to the two families $\left(\theta_{\lambda}\right)$ and $\left(\frac{1}{a} \theta_{\lambda}\right)$ which are biorthogonal with respect to the $L^{2}$-scalar product. We recall that the functions $\frac{1}{a(x)} \theta_{\lambda}(x)$, $\lambda \in \Lambda$, form a family of vaguelettes (see the end of the Section 3 ) and we proved that $\left(\int_{I} \theta_{\lambda}(x) d x\right)$ satisfy a Carleson condition. Thus, the inequalities $(7)$ and (8) hold. Finally, we proved that the collection of functions $\left(\theta_{\lambda}(x)\right)_{\lambda \in \Lambda}$ is a Riesz basis for $L^{2}(0,1)$, when completed with the Riesz basis $\left(v_{\gamma}^{\star}(x)\right)_{\gamma \in \Gamma_{0}}$ of 
$V_{0}$. That is, any function $f \in L^{2}(0,1)$ can be represented by the converging series in $L^{2}(0,1)$ as:

$$
f(x)=\sum_{\lambda \in \Lambda} \alpha_{\lambda} \theta_{\lambda}(x)+\sum_{\gamma \in \Gamma_{0}} \beta_{\lambda} v_{\gamma}^{\star}(x)
$$

where $\alpha_{\lambda}=\int_{0}^{1} f(x) \frac{1}{a(x)} \theta_{\lambda}(x) d x$ and $\beta_{\lambda}=\int_{0}^{1} f(x) \frac{1}{a(x)} v_{\gamma}^{\star}(x) d x$.

E3- We see that the proof of inequality (4) requires knowledge about the system biorthogonal to the basis $\left(u_{\lambda}(x)\right)_{\lambda \in \Lambda}$. Although we know that it exist, it is not sufficient because we must be able to estimate it. As it is shown in [13], the access to the biorthogonal system for $\left(u_{\lambda}(x)\right)_{\lambda \in \Lambda}$ is missing and this prevents us from proving (8) and hence also (3). In other words, it has not yet been proved that the analysis algorithm associated with $\left(u_{\lambda}(x)\right)_{\lambda \in \Lambda}$ is unconditionally stable. However, as was shown by (4), we know that the synthesis algorithm is unconditionally stable.

\section{$\S 6$ Final remarks}

The method treated in this chapter is not the only alternative for the use of Fourier techniques to construct the wavelets. To conclude this chapter, we want to make some remarks about an other approach, that was initiated by Meyer [10] and followed by Auscher, Cohen, Daubechies and Vial [2,5]. All this work is devoted to the construction of a basis of $L^{2}(I)$ consisting of Daubechies' compactly supported wavelets. Here, the main idea is to complete the set of wavelets with support inside the interval (which do not generate the whole $L^{2}(I)$ ) with a set of suitable functions. These functions show the advantage of being deduced from each other by dyadic dilation. The wavelets that are constructed in [10] do not satisfy any restrictive condition at the boundary of the interval. The construction is improved for numerical purposes in [5]. Finally, in [2] Auscher shows that one can prescribe boundary values for such wavelets. These wavelets also allow us to characterise the Sobolev spaces related to some homogeneous boundary value problem. For instance, consider the Dirichlet problem $-u^{\prime \prime}+u=f$ on $\left[0,+\infty\left[, f \in L^{2}[0,+\infty[\right.\right.$, $u(0)=0$ and $u(x) \rightarrow 0$ when $x \rightarrow+\infty$. This problem has a unique solution in $H^{2} \cap H_{0}^{1}=\left\{g \in H^{2}\left[0,+\infty[; g(0)=0\}\right.\right.$. In [2] a Riesz basis for $H^{2} \cap H_{0}^{1}$ is constructed. Notice that the restriction to the same interval of the wavelet basis constructed in E1 does not lead to such a basis (it only forms an orthonormal basis for $H_{0}^{s}[0,+\infty[, 0<s<2 m-2)$.

One of the most important issues in wavelet theory is to construct wavelet bases on open subsets of $\mathbb{R}^{n}$, which can be used for boundary value problems in partial differential equations. As we explained, this is already achieved on the interval (i.e., for $n=1$ ) where the simplicity of the geometry enables us to use a natural and simple construction. However, the extension to higher dimensional cases is 
much more difficult (the complexity of the geometry is only one of the additional difficulties). We expect that the flexibility of the general construction exposed in this chapter will lead to such desired results.

A last important issue is the development of adaptive wavelet decompositions. The construction of wavelets associated with a locally refined mesh of points, as is described in E3, was motivated by the development of adaptive schemes for the numerical approximation of the solution of partial differential equations in the presence of local singularities. The starting point of the construction in [13] was the adaptive algorithm for the periodic Burger equation, constructed by Liandrat and Tchamitchian [9]. This, however, is not the only direction to start investigations to apply wavelet research to the construction of adaptive codes for the numerical treatment of partial differential equations. Recently, much work has also been done in the field of adaptive multigrid methods, where the bases used for approximation are very much related to wavelet bases. It seems that a complete new field of research opens itself here at the interface of wavelet research and multigrid methods. We believe that this research area will generate many interesting results in the next few years.

Acknowledgments. Work for this paper by the second author was partially done while she was a guest at the University of Amsterdam, Faculty of Mathematics and Computer Science.

\section{References}

1. Auscher, P., Wavelets with boundary conditions on the interval, in Wavelets: $A$ Tutorial in Theory and Applications, C.K. Chui (ed.), Academic Press, Boston, 1992.

2. Auscher, P., Ondelettes à support compact et conditions aux limites, IRMAR, Rennes, 1992, preprint.

3. Auscher, P. and Ph. Tchamitchian, Ondelettes et conjecture de Kato, C. $R$. Acad. Sci. Paris 313 (1991), 63-66.

4. Auscher, P. and Ph. Tchamitchian, Conjecture de Kato sur les ouverts de $\mathbb{R}$, Rev. Mat. Iberoam. 8 (1992), 149-199.

5. Cohen, A., I. Daubechies, and P. Vial, Wavelets and fast wavelet transforms on the interval, AT\&T Bell-Laboratories, (1992), preprint.

6. Holschneider, M. and Ph. Tchamitchian, On the wavelet analysis of the Riemann's function, CPT-89/P.2274, Centre de Physique Théorique, Marseille, preprint.

7. Jaffard, S., Pointwise smoothness, two microlocalisation and wavelet coefficients, Publicacions Matemátiques 35 (1991), 155-168.

8. Jaffard, S. and Y. Meyer, Bases d'ondelettes dans les ouverts de $\mathbb{R}^{n}, J$. Math. pures et appl. 68 (1989), 95-108. 
9. Liandrat, J. and Ph. Tchamitchian, Resolution of the 1D regularized Burger equation using a spatial wavelet approximation algorithm and numerical results, ICASE Report, NASA, Hampton, Virginia, 1989

10. Meyer, Y., Ondelettes sur l'intervalle, Rev. Math. Iberoam. 7 (1991), 115-133.

11. Meyer, Y., Ondelettes et Opérateurs, Tome I: Ondelettes, Hermann, Paris, 1990.

12. Meyer, Y., Ondelettes et Opérateurs, Tome II: Opérateurs de Calderón Zygmund, Hermann, Paris, 1990.

13. Plantevin, F., Une application de la transformée continue en ondelettes à la mécanique quantique et Analyse multi-résolution adaptative, dissertation, Centre de Physique Théorique, CPT-92/P2815, Marseille, (1992).

Pieter W. Hemker

CWI

P.O. Box 4079

1009 AB Amsterdam

The Netherlands

pieth@cwi.nl

Frédérique Plantevin

Centre de Physique Théorique

CNRS - Luminy - case 907

13288 Marseille CEDEX 9

France

plantevi@cptvax.in2p3.fr 